\title{
Influence of 12-bit and 16-bit CT values of metals on dose calculation in radiotherapy using PRIMO, a Monte Carlo code for clinical linear accelerators
}

https://doi.org/10.1515/cdbme-2019-0150

\begin{abstract}
In this paper, the effect of computed tomography (CT) values of metals in 12-bit and 16-bit extended Hounsfield Unit (EHU) scale on dose calculations in radiotherapy treatment planning systems (TPS) were quantified. Dose simulations for metals in water environment were performed with the software PRIMO in 6MV photon mode. The depth dose profiles were analysed and the relative dose differences between the metals determined with 12-bit and 16-bit CT imaging, respectively, were calculated. Maximum dose differences of $\Delta \mathrm{Al}=3.0 \%, \Delta \mathrm{Ti}=4.5 \%, \Delta \mathrm{Cr}=6.2 \%$ and $\Delta \mathrm{Cu}=11.6 \%$ were measured. In order to increase the accuracy of dose calculation on patients with implants, CT imaging in the EHU scale is recommended.
\end{abstract}

Keywords: computed tomography, radiotherapy, PRIMO, Monte Carlo simulation

\section{Introduction}

The efficiency of radiotherapy treatment of patients with medical implants is reduced, particularly due to the limitations of computed tomography (CT) [1]. TPSs are based on CT data, providing tissue dependent information about their radiological properties, such as mass and electron density (ED). The majority of CT scanners used in radiology provide a conventional Hounsfield unit (CHU) scale, which suffices to properly represent human body tissues. HU values of high- $Z$ materials, like metallic implants, considerably exceed the maximum of the CHU scale, because these materials usually saturate at CHU's maximum. The EHU scale better reproduces the HU values of high- $\mathrm{Z}$ materials thus rendering itself appropriate for the purpose of dose calculations with TPS $[2,3]$.

\footnotetext{
${ }^{*}$ Corresponding author: Zehra Ese, Waldemar Zylka, Department of Electrical Engineering and Applied Natural Science, Westphalian University, Campus Gelsenkirchen, Germany, e-mail: zehra.ese@studmail.w-hs.de

${ }^{*}$ Corresponding author: Zehra Ese, Department of General and Theoretical Electrical Engineering, University of DuisburgEssen and CENIDE - Center of Nanointegration Duisburg-Essen, Bismarckstr. 81, 47048 Duisburg, Germany
}

The purpose of this study is to examine the effect of extended bit imaging of metals in dose calculations using PRIMO software. PRIMO simulate clinical linear accelerators to estimate dose distributions. It is based on PENELOPE, a Monte Carlo code for the calculation of photon and electron transport. A water phantom containing various metals were simulated. The dose simulations are based on CT images reconstructed in the $\mathrm{CHU}$ and EHU scales. The results are compared to each other.

\section{Material and Methods}

Definition of Hounsfield scales. In CT imaging the X-ray attenuation coefficients $\mu$ of the irradiated tissues are reconstructed into a matrix of equally sized voxels. Usually the attenuation coefficient of a particular image voxel is expressed in Hounsfield units, known as CT values, using the linear function

$$
H(\mu)=\left(\frac{\mu}{\mu_{w}}-1\right) 1000 \mathrm{HU},
$$

where $\mu_{w}$ is the attenuation coefficient of water. This general definition does not limit the range of values of the function $H(\mu)$. Despite of (1), CT vendors use technically suitable, but limited HU scales when storing CT measurements in DICOM files. In radiology, the HU scale $[-1024 \mathrm{HU} ;+3071 \mathrm{HU}]$, which uses 12-bits, is a de-facto standard. This CHU scale is suitable for the imaging of human body tissue, but is limited for high- $Z$ materials. In a previous study, we showed that high-Z materials are incorrectly mapped to the CHU maximum causing potential errors in dose calculations [2,3]. In TPS, the calculation of dose distribution is based on HU values from CT images [4]. To this end, all CT values must be converted into electron density (ED) using a calibration function obtained from the CT scale in use, e.g. CHU. This renders the calculation of the ED of high-Z materials incorrect. CT vendors offer extended HU scales (EHU) which allows a correct mapping of high-Z materials [3]. To represent various HU scales in a unified form, Eq (1) is rewritten in terms of properties stored in DICOM files:

$$
H(\mu)=s \beta+i,
$$


where $\beta=1000 \mu / \mu_{w}$ is a dimensionless quantity representing the measured attenuation coefficient, $s$ is the slope and $i$ the intercept, both expressed in HU units, of the linear function $H(\mu)$ mapping attenuation coefficients $\mu$ to Hounsfield values. Specific values for $s, i$ and the resolution (minimal increaement) $\Delta H$ for the different HU scales are given in Tab. 1 . The number of HU values available in a CT scanner is limited solely by the bits that are provided for each voxel.

Tab. 1: Definition of parameters in Eq. (2) for various HU scales.

\begin{tabular}{lrrrc}
\hline HU scale & $s$ [HU] & $i$ [HU] & $\Delta H$ & \# values \\
\hline Equation (1) & 1 & -1000 & 1 & $\infty$ \\
CHU, 12 bit & 1 & -1024 & 1 & $2^{12}$ \\
EHU, 12 bit & 10 & -10240 & 10 & $2^{12}$ \\
EHU, 16 bit & 1 & -32768 & 1 & $2^{16}$ \\
\hline
\end{tabular}

\subsection{CT acquisition of metals}

For the dose simulations, a cube-shaped water tank model with an edge length of $350 \mathrm{~mm}$ was generated in Matlab (version R2018b). In total, 256 CT scans with $256 x 256$ pixels per image were generated. The overall reconstruction results in cubic voxels with a length of $1.37 \mathrm{~mm}$ and a resolution of 0.73 $\mathrm{pixel} / \mathrm{mm}$. The origin of the coordinate system is located at the center of the upper surface of the water tank as shown in Fig. 1. In $10 \mathrm{~mm}$ depth a cylinder, made of a certain material, was created with a diameter of $21 \mathrm{~mm}$ and a hight of $5 \mathrm{~mm}$. Five CT scans were generated corresponding to the five metallic materials used for the cylinder as listed in Tab.2.

Tab. 2: Physical properties of the metal objects aluminium (Al), titanium (Ti), chromium $(\mathrm{Cr})$ and copper $(\mathrm{Cu})$, which are positioned within the water phantom for dose simulation. The EDs are calculated relative to $\mathrm{ED}_{w}=3.343 \mathrm{~cm}^{-3}$ (ED of water).

\begin{tabular}{lccccc}
\hline Material & $\mathbf{Z}$ & $\rho\left[\mathrm{g} / \mathrm{cm}^{3}\right]$ & rel ED & HU & HU scale \\
\hline $\mathrm{Al}$ & 13 & 2.70 & 2.34 & 2729 & EHU, 16 bit \\
$\mathrm{Ti}$ & 22 & 4.50 & 3.73 & 4433 & EHU, 16 bit \\
$\mathrm{Cr}$ & 24 & 7.14 & 5.94 & 7156 & EHU, 16 bit \\
$\mathrm{Cu}$ & 29 & 8.92 & 7.33 & 8876 & EHU, 16 bit \\
$\mathrm{Cu}$ & 29 & 8.92 & 7.33 & 3071 & $\mathrm{CHU}, 12$ bit \\
\hline
\end{tabular}

The $\mathrm{HU}$ values of $\mathrm{Al}, \mathrm{Ti}, \mathrm{Cr}$ and $\mathrm{Cu}$ were determined experimentally using CT imaging with a Siemens Somatom Force and GE LightSpeed RT CT at $120 \mathrm{kV}$ and $230 \mathrm{~mA}$ and using a convolution kernel $\mathrm{Br} 40$. All objects were coin shaped with a the same geometry as chosen on the artificial CT scan.
The HU values are quantified following the method published in [3]. The HU values of the metals and the mass and relative electron density to water are given in Tab. 2 .

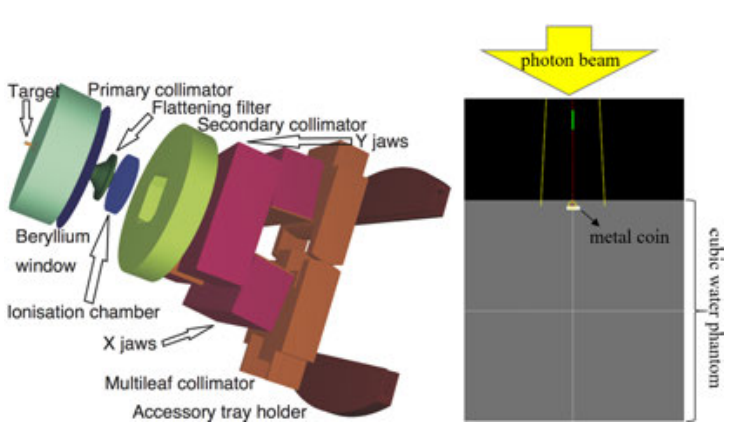

Fig. 1: The simulated linac geometry of a Varian Clinac 2100 $\mathrm{C} / \mathrm{D}$ operating in photon mode in PRIMO environment [7] (left).; PRIMO dose simulation configuration showing phantom construction in central axial plane with the illustration of the radiation field size and position (right).

\subsection{Dose simulation in PRIMO}

Dose simulations for metals in water environment were performed with PRIMO (version 0.3.1.1681). PRIMO is a software in which clinical linear accelerators (linac) are simulated and absorbed dose distributions in CT studies and phantoms are estimated [5]. PRIMO is based on PENELOPE, penEasy, penEasyLINAC and a graphical user interface that combines all components. PENELOPE is a Monte Carlo code for the simulation of coupled electron and photon transport [6]. PenEasy is a main program for PENELOPE that includes several source models, tallies, variance-reduction techniques and the possibility of combining quadric and voxelized geometries $[6,7]$. penEasyLINAC is a complementary tool that generates the input files required for the simulation of linacs with PENELOPE [7]. In PRIMO, the simulation is segmented into three parts, named s1, s2 and s3. The first segment is the linac head. Segment 2 is defined as the constructive part, including collimators, jaws and MLCs as shown in Figure 1. The geometric region (s3) corresponding to the patient or phantom, in which the absorbed dose is estimated. The simulation of $\mathrm{s} 1$ and $\mathrm{s} 2$ is stored in a phase-space file. This file stores the state of particles (energy, position, direction of flight) in traversed plane [7].

In this study a Varian Clinac 2100, one of the linac models implemented in PRIMO, were simulated. Figure 1 shows the schematic construction of this linac operating in photon mode. The DICOM CT images are imported in PRIMO. The GE LightSpeed RT CT scanner calibration curve has been im- 


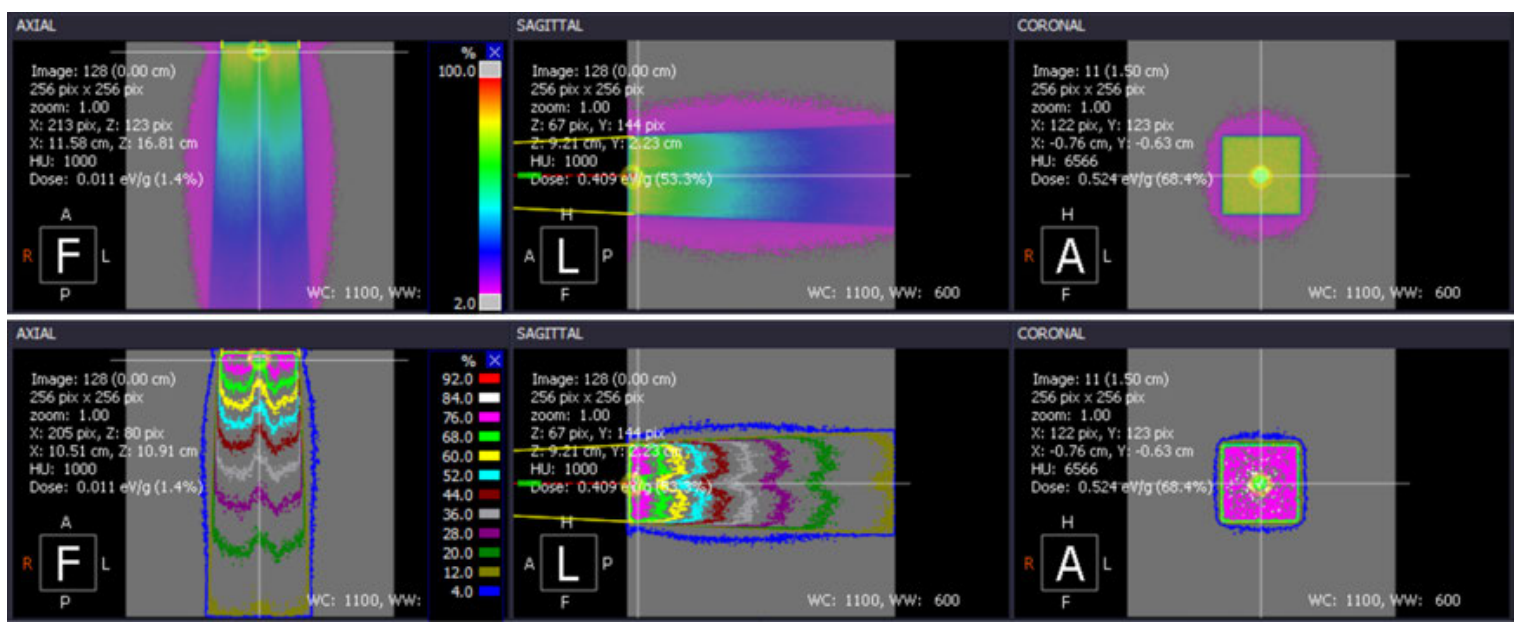

Fig. 2: Dose distribution estimated for the simulation of segments $s 2$ and $s 3$. The absorbed dose distribution at the central plane of the phantom in axial, sagital and coronal view is shown, as color wash map (top line) and isodose curve map (lower line).

ported, in order to assign the material densities to $\mathrm{CT}$ values. Previously, the calibration curve were derived with extended Hounsfield Unit scale [3]. For the simulation a photon beam energy of $6 \mathrm{MV}$ and a static radiation field of $10 \mathrm{~cm} \times 10$ were chosen at the surface of the metal object, with a SSD of $100 \mathrm{~cm}$ (see Fig.1). PRIMO reports absorbed doses in units of eV/g per primary particle. The Monte Carlo Code penEasy/PENELOPE is used for the simulation. A splitting factor of 300 were chosen in order to reduce variance. Since, the simulation of a particular linac head results in the same phase-space file, segment 1 were simulated only once and the resulting phase-space file were linked to the other simulations.

The dose distributions estimated for $\mathrm{s} 2$ and $\mathrm{s} 3$ were analysed (see Fig.2) for each simulation. The depth dose curves (DDC) at the central part of the beam were derived for each CT scan containing a different metal object. In particular, we compare the DDCs for various metals with $\mathrm{HU}$ values determined in EHU as well as the same material measured in $\mathrm{CHU}$ and EHU scale.

\section{Results}

The absorbed dose profiles, derived from the PRIMO dose distribution maps are shown in Figure 3. The DDCs were measured at the isocenter. The DDC for each metal are represented in a different color in Figure 3. The maximum absorbed dose for each simulation occur in front of the object surface. A dose built-up effect is seen at this level. Figure 3 shows an enlarged view of the first two $\mathrm{cm}$ depth (marked with Z1). It can be observed that the build-up effect is more pronounced with increasing material density. At this level, copper show a $10 \%$ higher absorbed dose as compared to aluminium.
Immediately after penetration of the sample, the dose drops sharply. The strength of the effect depends also on the mass density. The higher the density of a material, the higher its absorption rate. The depth dose progression for the metals $\mathrm{Al}, \mathrm{Ti}, \mathrm{Cr}$ and $\mathrm{Cu}$, measured with $\mathrm{CT}$ values given by the EHU scale, are compared to the dose profile of $\mathrm{Cu}$ measured using CT values from the $\mathrm{CHU}$ scale. When using the $\mathrm{CHU}$ values, the maximum possible $\mathrm{HU}$ value for all metals used in this study was $+3071 \mathrm{HU}$. Therefore, only the dose simulation for copper was simulated with the $\mathrm{CHU}$ value. Maximum dose differences of $\Delta \mathrm{Al}=3.0 \%, \Delta \mathrm{Ti}=4.5 \%, \Delta \mathrm{Cr}=6.2 \%$ and $\Delta \mathrm{Cu}=11.6 \%$ were determined. Figure 3 (upper diagramm) show the transversal absorbed dose distribution at 12 $\mathrm{mm}$ depth. The same effects and dose distributions are seen.

\section{Discussion and Conclusion}

The EHU scale better reproduces the HU values of highdensity materials and has no effect on the $\mathrm{HU}$ values of normal tissue [5]. Therefore, the EHU scale is more suitable for the purpose of dose calculation in radiotherapy for patients with and without metallic implants. However, the EHU scale does not improve the image quality of CT images. Image artifacts caused by metals are still present and require additional optimization e.g. by metal artifact reduction (MAR) algorithms [9]. Since MAR algorithms can falsify the HU values they should be used with care.

PRIMO is based on different calculation algorithms and is not a clinically used TPS tool. In this study, the absorbed dose values were given in $\mathrm{eV} / \mathrm{g}$. In order to make them comparable with real data, these values can be converted [7]. The effect of dose calculation for metals with $\mathrm{CHU}$ and EHU values was 

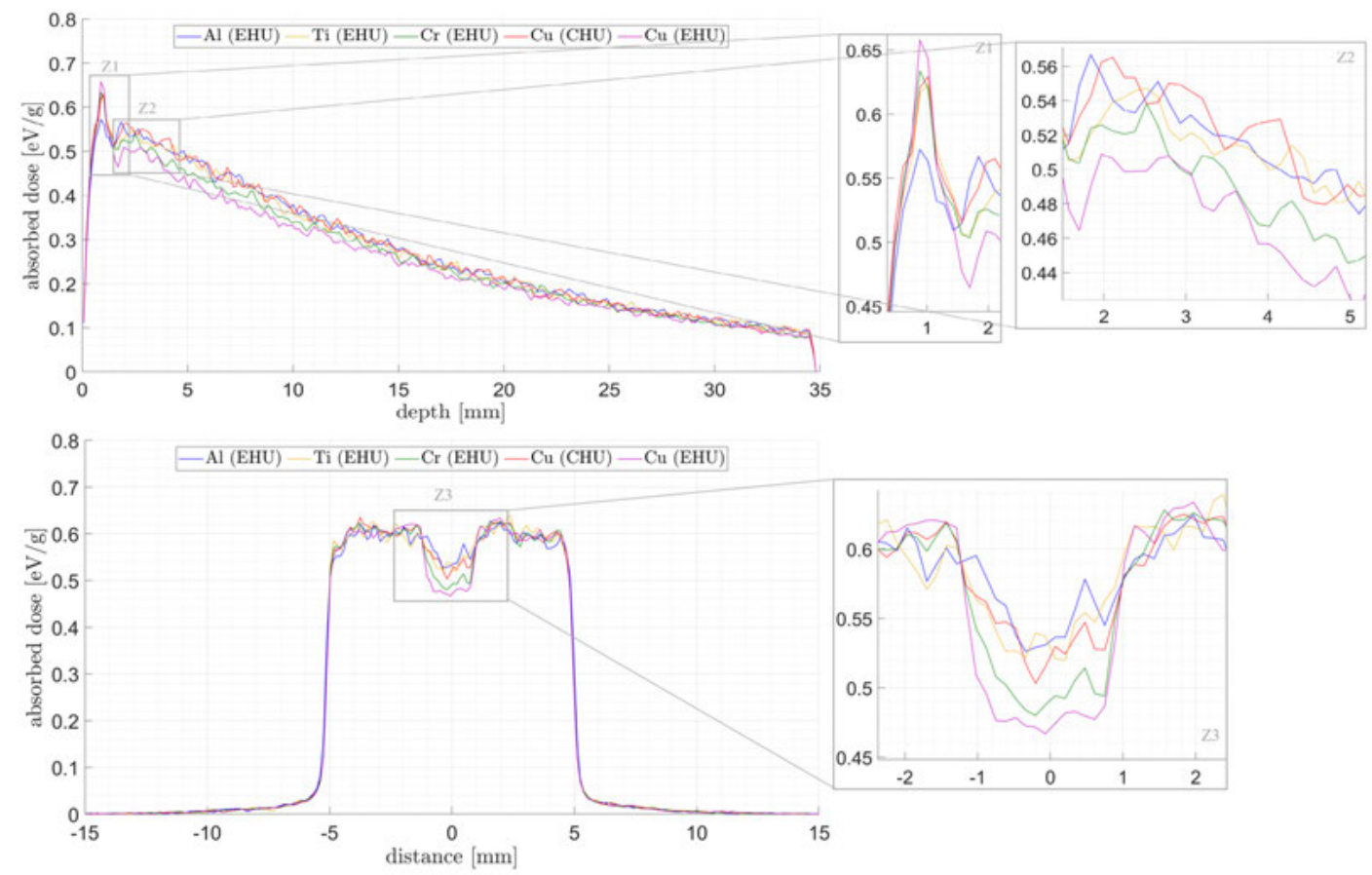

Fig. 3: Depth dose profiles measured at the center of the phantom for each metal (top line). Copper were simulated twice, for the CT data acquired at the $\mathrm{CHU}$ and $\mathrm{EHU}$ scale. The transversal dose profile in $12 \mathrm{~mm}$ depth is shown in the upper diagram.

clarified. The dose is underestimated when using CHU scale values for metals. The higher the mass density of a material the higher the difference in dose calculation for the two HU scales. It is expected that the dose differences increase with increasing photon beam energy. Therefore, further investigations will be carried out to quantify this effect as a function of the radiation energy. Also in-vitro measurements will be performed to validate the simulation results.

Medical electronic implants may malfunction when exposed to radiation. In order the occurrence of malfunction as low as possible, a dose limit of $2 \mathrm{~Gy}$ on the implants was recommended by the AAPM Task group. No 34 [9]. Dosimetry on implants is still difficult and TPS underestimates the total dose, as shown in this paper. Imaging with an extended HU scale leads to a more accurate dose estimation at the implant and thus increases patient safety.

\section{Author Statement}

Research funding: The author state no funding involved. Conflict of interest: Authors state no conflict of interest.

\section{References}

[1] Tang X, Changran G, Han W, Shu D, Hou X, Chen D. Dosimetry effects of metal implants in patient body during radiation therapy.Journal of Nanjing University of Aeronautics and Astronautics. 2013 45(6):819-823.

[2] Ese Z, Kressmann M, Kreutner J, Schaefers G, Erni D, Zylka $W$. Influence of conventional and extended CT scale range on quantification of Hounsfield units of medical implants and metallic objects. tm. 2018; 85(5):343-350.

[3] Ese Z, Qamhiyeh S, Kreutner J, Schaefers G, Erni D, and Zylka W. CT Extended Hounsfield Unit range in radiotherapy treatment planning for patients with implantable medical devices. Springer Nature Singapore Pte Ltd., IFMBE Proc. 2019;68(3),599-603.

[4] Schneider U, Pedroni E, Lomax A. The calibration of CT hounsfield units for radiotherapy treatment planning. Phys Med Biol. 1996;41:111-124.

[5] Rodriguez M, Sempau J, Brualla L. PRIMO : A graphical environment for the Monte Carlo simulation of Varian and Elekta linacs. Strahlenther. Onkol. 2013;189:881-6.

[6] Brualla L, Rodriguez M, Sempau J and Andreo P. PENELOPE/ PRIMO-calculated photon and electron spectra from clinical accelerators. Radiation Oncology 2019;14(6):3-10.

[7] Brualla L, Rodriguez M, Sempau, J. PRIMO user's manual software version 0.3.1.1600. www.primoproject.net. 2018.

[8] Giantsoudi D, De Man B, Verburg J et al. Metal artifacts in computed tomography for radiation therapy planning: dosimetric effects and impact of metal artifact reduction. Phys Med Biol 2017; 62 (8): R49-R80.

[9] Marbach JR, Sontag MR, Van Dyk J, Wolbarst AB. Management of Radiation Oncology Patients with Implanted Cardiac Pacemakers: Report of AAPM Task Group No.34. Medical Physics 1994;21(1): 85-90 